\title{
Early Therapeutic Penetrating Keratoplasty for Fungal Keratitis
}

\author{
Yusuf Kocluk $^{1 *}$, Mehmet Cubuk ${ }^{1}$, Emine Alyamac Sukgen ${ }^{1}$, Nevzat Unal ${ }^{1}$, Selim Cevher ${ }^{1}$ \\ ${ }^{1}$ Adana Numune Training and Research Hospital, Department of Ophthalmology, Adana, Turkey.
}

\begin{abstract}
Background: To present two cases who developed fungal keratitis after an organic trauma and who were treated with therapeutic penetrating keratoplasty in the early period.

Case presentations: A 14 years old female and a 63 years old male patients came with the complaints of pain after organic trauma. Fungal keratitis was diagnosed in both two cases. TPKP was performed on the $5^{\text {th }}$ day of the topical antifungal treatment (Case 1), on the $5^{\text {th }}$ day of the topical antifungal treatment (Case 2) and $7^{\text {th }}$ day of the trauma (both two cases). Graft transparency was protected in the post-operative period; no recurrence or fungal reactivation was detected in two patients.

Conclusion: The study has proven that TPKP performed in the early period of fungal keratitis enables rapid infection eradication and rapid visual success.
\end{abstract}

Key words: Fungal keratitis; Early Therapeutic Penetrating Keratoplasty; Organic trauma

\section{Introduction}

The most common types of fungal keratitis, which is one of the most frightening diseases in ophthalmology, are filamentous fungi (Fusarium types, Aspergillus types) and yeast fungi (Candia types, Cryptococcus types). Filamentous species were the most common causative pathogens (1). Most studies report that traumas caused by plant and organic material are the most common causes of fungal keratitis. Other risks include use of contact lens, use of topical steroids and antibiotics, corneal surface diseases (herpes keratitis, keratitis sicca, atopic keratoconjunctivitis, recurrent erosion), eye surgery (reactive surgery, keratoplasty, loose corneal sutures), immune suppression, and diabetes (2). Early stages of the infection might be controlled by appropriate antifungal agents, but therapeutic penetrating keratoplasty (TPKP) is the treatment of choice for intractable cases (3).

*Corresponding Author: Yusuf Kocluk; Adana Numune Training and Research Hospital, Dept. of Ophthalmology, Adana, Turkey. E-mail: kocluk99@gmail.com Received: Apr 21, 2016 Accepted: May 10, 2016 Published Online: May 13, 2016.
In most cases after TPKP the pathogen is successfully eradicated by surgery and the development of endophthalmitis, orbital cellulitis, or even panophthalmitis is avoided.

This study aims to present two cases who developed fungal keratitis after an organic trauma and who were treated with TPKP in the early period.

\section{Case 1}

The fourteen-years old female patient came with the complaints of pain, redness, and reduced vision after something got into her left eye while working in the field two days before. Visual acuity (VA) was 0.0001 in the left eye. Intraocular pressure (IOP) was normal digitally. In the central left eye cornea, there was suppurative lesion, corneal thinning, and hypopyon (Figure 1a). Posterior segment was normal by ultrasound examination.

This is an Open Access article distributed under the terms of the Creative Commons Attribution Non-Commercial License (http://creativecommons.org/licenses/bync/4.0/) which permits unrestricted non-commercial use, distribution, and reproduction in any medium, provided the original work is properly cited. 
Corneal scrapings obtained were inoculated onto $5 \%$ blood agar, EMB (Eosin Methylene Blue) agar, chocolate agar, and SDA (Sabouraud dextrose agar) media. Plenty of hyphal was observed in the direct microscopic examination. Fungal keratitis was diagnosed. The treatment was started with Fortified vancomycin $(50 \mathrm{mg} / \mathrm{ml})$, Fortified ceftazidime $(50$ $\mathrm{mg} / \mathrm{ml}$ ), and amphotericin B $0,15 \%$ in hourly doses.

Natamycin pomade was not given because it is not available in our country. No reduction in the size of the lesion was detected on the 4th day of the treatment (Figure 1b). On the contrary, due to the extension of infiltrates through limbus, topical voriconazole was aimed, but it could not be obtained. Then, TPKP was performed with the patient under general anesthesia on the 7 th day of the trauma and on the $5^{\text {th }}$ day of the topical amphotericin B $0,15 \%$ treatment.

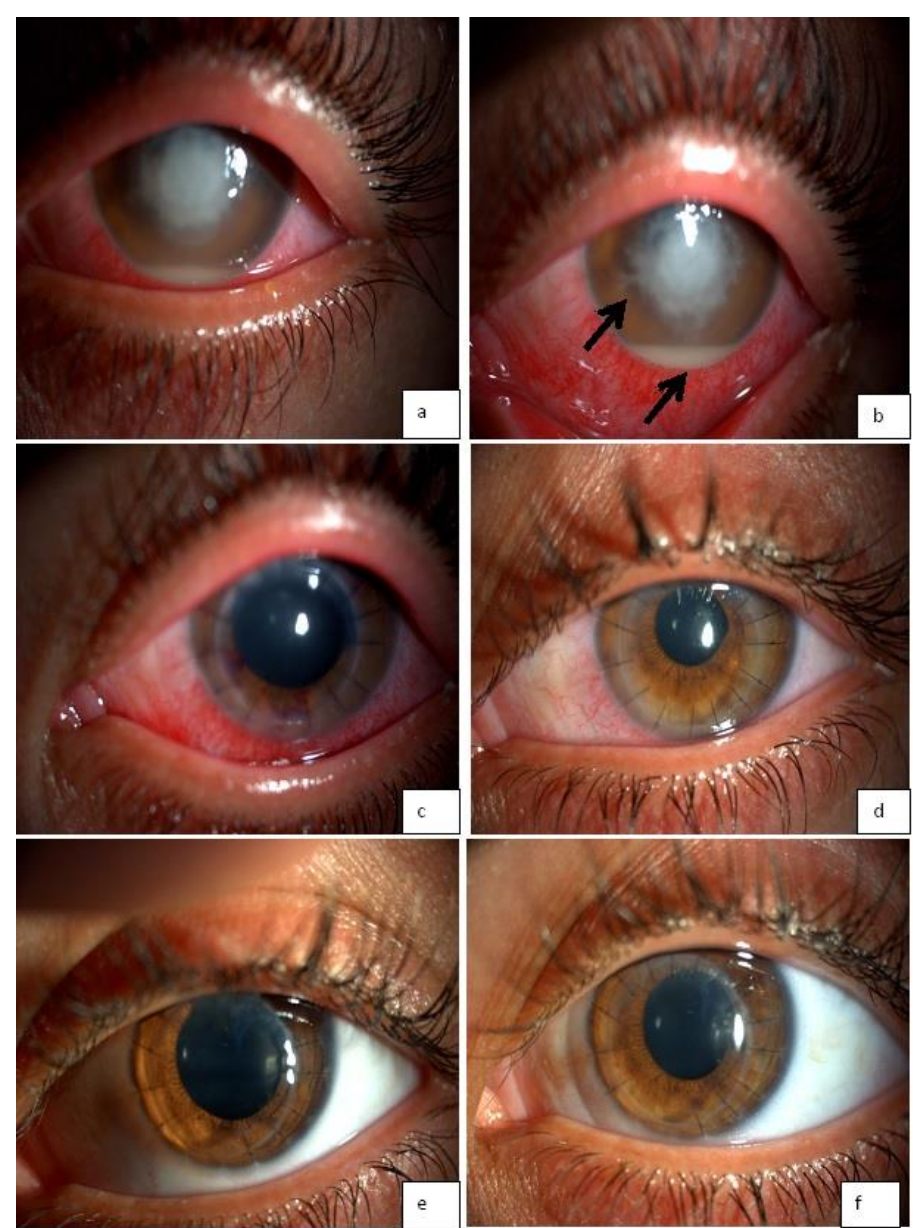

Figure 1. Figures of case 1 (a) arrival at the hospital, before topical amphotericin B treatment, (b) Five days after topical amphotericin B, before surgery, an increase of hypopyon and infiltrate, (c) first day after TPKP, (d) one month after TPKP, (e) three months after TPKP, (f) nine months after TPKP (TPKP: Therapeutic penetrating keratoplasty).
Excision of the recipient cornea was performed with $7.75 \mathrm{~mm}$ vacuum trepan in the size involving all infective corneas. The membranes covering the iris surface and angle in anterior chamber were removed. The tissue, which was excised was sent for microbiology evaluation. Anterior chamber and margin of the recipient cornea were washed with amphotericin B $(0.05 / 1 \mathrm{cc})$. Then, a new operation table and set were prepared; $8.25 \mathrm{~mm}$ size donor cornea was sutured to the recipient bed, one by one and using 10/0 nylon suture. $0,005 \mathrm{mg} / 0,1 \mathrm{cc}$ amphotericin B was performed by intracameral.

Following the operation, acetazolamide $250 \mathrm{mg}$ tablet (twice a day), topical moxifloxacin (six times a day), topical amphotericin B $0,15 \%$ (eight times a day), artificial tears (eight times a day), topical cyclosporine $0,05 \%$ drop (four times a day) methylprednisolone tablet $32 \mathrm{mg} /$ day, lansoprazole tablet (once a day) was started.

There was culture growth in the post-operative period. Type description of the strain was performed using VITEK ${ }^{\circledR}$ MS MALDI-TOF (Matrix assisted laser desorption ionization time of flight mass spectrometry) version 2.0 system. Type description was reported 99.4\% as Fusarium solani (Figure 2 a-d).
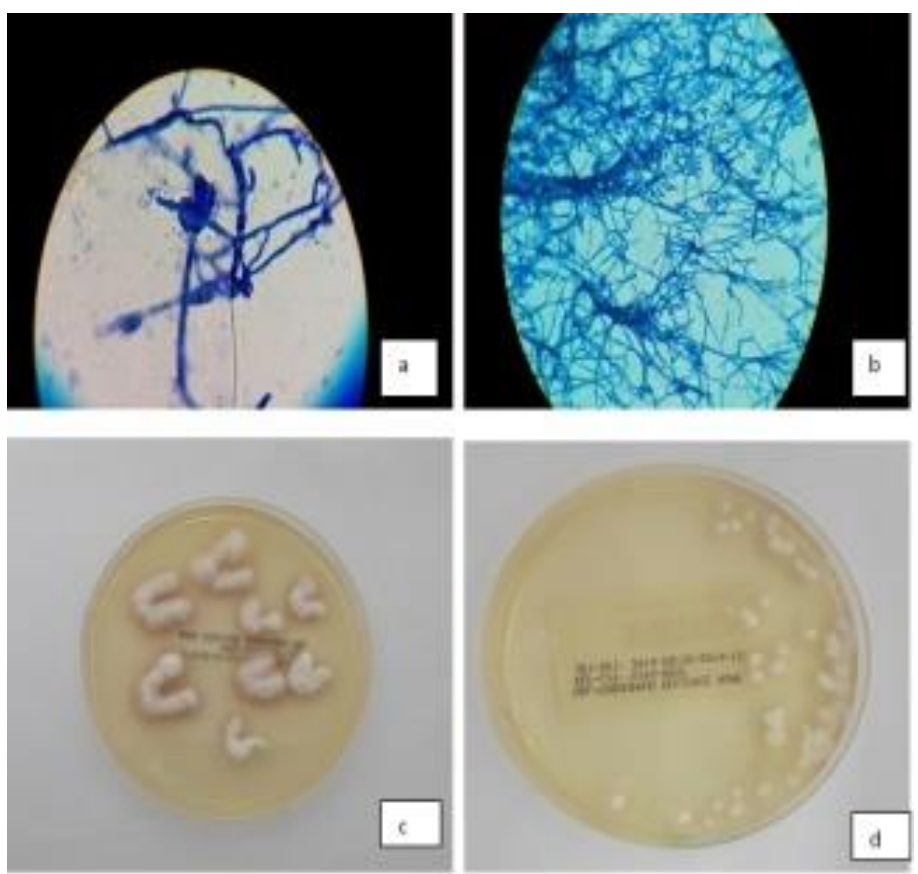

Figure 2. Staining by methylene blue of Fusarium solani (a, b) and identification by Matrix assisted laser desorption ionization time of flight mass spectrometry, culture media (c, d).

Moxifloxacin, methylprednisolone (in tapering doses) and lansoprazole tablet was stopped in the third post- 
operative week. Dexamethasone drops (two times a day) was included in the treatment and continued in increasing doses. Topical amphotericin B 0,15\% was continued in tapering doses until the third post-operative month.

Graft transparency was found to continue until the $9^{\text {th }}$ month of the follow-ups, no recurrence or fungal reactivation was detected (Figure 1c-f). Best corrected visual acuity (BCVA) was 0.8 in the postoperative $9^{\text {th }}$ month; and IOP was normal.

\section{Case 2}

63 years-old male patient came to the clinic with the complaint that a walnut fell on his left eye a day before. VA was 0.03 in the left eye. IOP was normal digitally. In the central left eye cornea, there was infiltrative lesion, corneal thinning, and hypopyon (Figure 3a). Posterior segment was normal on ultrasound examination. Fungal was positive in direct microscopic examination, but no growth was displayed in culture.
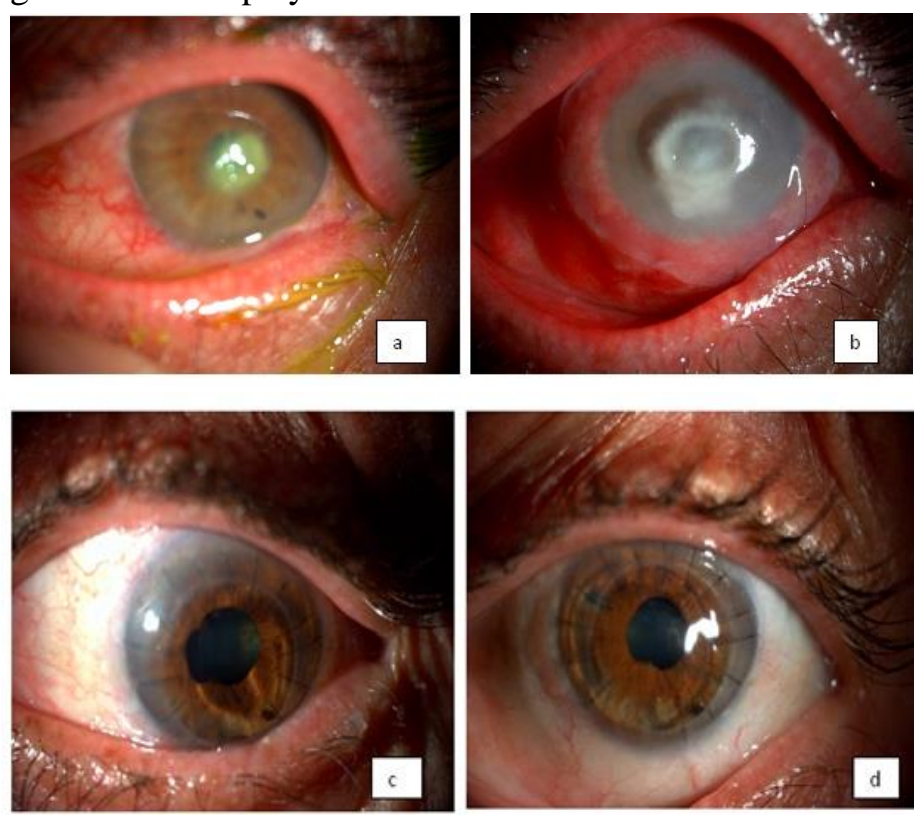

Figure 3. Figures of case 2 (a) First day after trauma and before treatment, (b) 4 days after topical, intracameral voriconazole treatment, and AMT, before TPKP, (c) 1 month after TPKP, (d) 11 months after TPKP (AMT: Amniotic membrane transplantation, TPKP: Therapeutic penetrating keratoplasty)

The treatment was started with hourly doses of Fortified vancomycin $(50 \mathrm{mg} / \mathrm{ml})$, Fortified ceftazidime (50 $\mathrm{mg} / \mathrm{ml}$ ), amphotericin B $0,15 \%$. However, because there was no response to the present treatment and progress, the antifungal was changed on the second day. Topical voriconazole (1\%) was given hourly. Besides, intrastromal and intracameral $(0.1 \mathrm{mg} / 0.1 \mathrm{cc}$ doses $)$ voriconazole, amniotic membrane transplantation (AMT) was applied due to central corneal thinning. There was no response to the treatment (Figure 3b). TPKP was performed under local anesthesia on the 4th day of the topical voriconazole treatment and $7^{\text {th }}$ day of the trauma.

Differently from Case 1, preoperative anterior chamber and recipient corneal margin was washed with voriconazole $(0.1 \mathrm{mg} / 0.1 \mathrm{cc})$ and $0,1 \mathrm{mg} / 0,1 \mathrm{cc}$ voriconazole intracameral was performed. Medication in the post-operative period was done similarly to the medication in Case 1. Graft transparency was protected in the post-operative period; no recurrence or fungal reactivation was detected (Figure 3c, d). BCVA was 0.5 with a grade 2 nuclear sclerosis in the postoperative $11^{\text {th }}$ months; and IOP was normal.

\section{Discussion}

Corneal fungal infections form between 6 to $53 \%$ of ulcerative keratitis. Fungal keratitis are generally related with traumas caused by plants or metals contaminated with fungi; and they are generally formed through the medium of filamentous fungi. Trauma can be too weak for the patient to remember (2). In both cases the present study had an organic trauma history.

In India, 10.7\% out of 264 fungus keratitis were found to have Fusarium solani (4). The Fusarium proportion was found between 45 and $67 \%$ in studies conducted in Brazil (5). The present study has also detected Fusarium in Case 1, but no culture growth was displayed in the other case.

If there is hyphae and filamentous fungus growth in culture, topical $5 \%$ natamycin seems to be the optimal medicine, and topical $0.15 \%$ amphotericin-B is an alternative. As natamycin is not available in our country, amphotericin-B was used as the first option in this study. Surgery is needed in cases which show progress despite maximum antifungal treatment, which have an extension tendency to limbus, and which have perforation threat. In a study conducted in Brazil, 22 cases out of 41 (54\%) needed PKP; and 19 of these 22 cases were observed to have a perforation and 3 of them had no response to the medical treatment (5). Oechsler et al. Investigated 52 
patients with fungal keratitis, performed PKP in 7 patients, and detected fusarium in all these 7 patients. Of these 7 patients, 4 of them were found to have a perforation and 3 of them gave no response to the medical treatment (6). In the present study early surgical intervention was preferred for both cases because of severe pain, no response to the treatment, and the tendency of infiltrates to extend to the limbus.

Use of corticosteroid in the post-operative period requires a great deal of attention in order to prevent graft reaction. Inflammation of corticosteroid infection or the probability of causing super infection should be kept in mind. Perry et al. reported that use of cyclosporine-A after PKP $(0.5 \%)$ is a good alternative to corticosteroid (7). In this study, topical steroid was not used in the first three post-operative weeks. Later on, it was applied in increasing doses and under the umbrella of a topical antifungal. Topical cyclosporine-A $(0.05 \%)$ was used as of the first post-operative day.

Recent studies have reported that early surgery performed without waiting perforation and the extension of the lesion to limbus has better outcomes in resistant cases $(8,9)$. Rapid visual success, symptomatic relief, and shorter hospital stay have been achieved by the early surgery intervention in the cases in this study. Both cases were discharged from the hospital on the third postoperative day.

\section{Conclusion}

Consequently, with the two cases diagnosed with fungal keratitis, the present study has proven that TPKP performed in the early period enables rapid infection eradication and rapid visual success.

\section{Acknowledgements}

The authors did not receive any financial support from any public or private sources. The authors have no financial or proprietary interest in a product, method, or material described herein.

\section{References}

1. Ho JW, Fernandez MM, Rebong RA, Carlson AN, Kim T, Afshari NA Microbiological profiles of fungal keratitis: a 10year study at a tertiary referral center. J Ophthalmic Inflamm Infect 2016; 6: 5.

2. Klotz SA, Penn CC, Negvesky GJ, Butrus SI. Fungal and parasitic infections of the eye. Clin Microbiol Rev 2000; 13: 662-685.
3. Xie L, Zhai H, Shi W. Penetrating keratoplasty for corneal perforations in fungal keratitis. Cornea 2007; 26: 158-162.

4. Rautaraya B, Sharma S, Kar S, Das S, Sahu SK. Diagnosis and treatment outcome of mycotic keratitis at a tertiary eye care center in eastern India. BMC Ophthalmol 2011; 11: 39.

5. Oechsler RA, Yamanaka TM, Bispo PJ, Sartori J, Yu MC, Melo AS, Miller D, Hofling-Lima AL. Fusarium keratitis in Brazil: genotyping, in vitro susceptibilities, and clinical outcomes. Clin Ophthalmol 2013; 7: 1693-1701.

6. Rafael A Oechsler, Michael R Feilmeier, Darlene Mille. Fusarium keratitis: genotyping, in vitro susceptibility and clinical outcome. Cornea 2013; 32: 667-673.

7. Perry HD, Doshi SJ, Donnenfeld ED, Bai GS. Topical cyclosporine $\mathrm{A}$ in the treatment of therapeutic keratoplasty for mycotic keratitis. Cornea 2002; 21: 161-163.

8. Sharma N, Jain M, Sehra SV, Maharana P, Agarwal T, Satpathy G, Vajpayee RG. Outcomes of therapeutic penetrating keratoplasty from a tertiary eye care centre in northern India. Cornea 2014; 33(2): 114-118.

9. Garq P, Roy A, Rof S. Update on fungal keratitis. Curr Opin Ophthalmol 2016; 27: 0-0(epub).

How to cite?

Kocluk Y, Cubuk M, Sukgen EA, Unal N, Cevher S. Early therapeutic penetrating keratoplasty for fungal keratitis. J Immunol Clin Microbiol 2016; 1(1): 18-21.

DOI: http://dx.doi.org/10.5455/jicm.3.20160510.

Submit your next manuscript to the JICM and take full advantage of;

- Convenient online submission,

- Thorough peer review, Fast Response,

- No space constraints or color figure charges,

- Immediate publication on acceptance,

- Inclusion in Scopemed and High quality indexes,

- Research which is freely available for redistribution.

To submit your manuscript, please click on http://www.jiacm.com

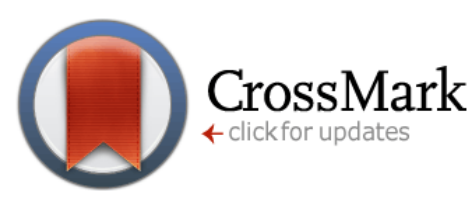

Published by The QMEL.org International Medical Education Library

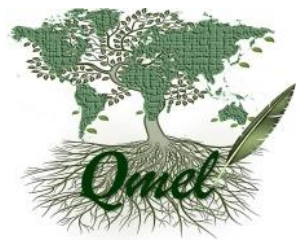

